\title{
Polarization and depolarization of monomial ideals with application to multi-state system reliability
}

\author{
Fatemeh Mohammadi ${ }^{1}$ (D) Patricia Pascual-Ortigosa ${ }^{2}$ (D) \\ Eduardo Sáenz-de-Cabezón ${ }^{2}$ (D) Henry P. Wynn ${ }^{3}(\mathbb{D}$
}

Received: 20 July 2018 / Accepted: 19 April 2019 / Published online: 21 May 2019

(c) Springer Science+Business Media, LLC, part of Springer Nature 2019

\begin{abstract}
Polarization is a powerful technique in algebra which provides combinatorial tools to study algebraic invariants of monomial ideals. We study the reverse of this process, depolarization which leads to a family of ideals which share many common features with the original ideal. Given a squarefree monomial ideal, we describe a combinatorial method to obtain all its depolarizations, and we highlight their similar properties such as the graded Betti numbers. We show that even though they have many similar properties, their differences in dimension make them distinguishable in applications in system reliability theory. In particular, we apply polarization and depolarization tools to study the reliability of multistate coherent systems via binary systems and vice versa. We use depolarization as a tool to reduce the dimension and the number of variables in coherent systems.
\end{abstract}

Keywords Monomial ideals · Polarization · Depolarization · Algebraic reliability

Eduardo Sáenz-de-Cabezón

eduardo.saenz-de-cabezon@unirioja.es

Fatemeh Mohammadi

fatemeh.mohammadi@bristol.ac.uk

Patricia Pascual-Ortigosa

papasco@unirioja.es

Henry P. Wynn

h.wynn@1se.ac.uk

1 School of Mathematics, University of Bristol, Bristol, UK

2 Departamento de Matemáticas y Computación, Universidad de La Rioja, Logroño, Spain

3 Department of Statistics, London School of Economics, London, UK 


\section{Introduction}

\subsection{Background}

Polarization is an operation that transforms a monomial ideal into a squarefree monomial ideal in a larger polynomial ring, preserving several important features of the original ideal such as the graded Betti numbers. The main idea behind polarization is the possibility of using the combinatorial properties of squarefree monomial ideals when studying problems about general monomial ideals. Polarization is used in a wide variety of applications in the theory of monomial ideals. For example, it was used by Hartshorne to prove the connectedness of the Hilbert scheme by showing that distractions of ideals can be described as specializations of polarizations of monomial ideals [17]. One of the main applications is its use to study the Cohen-Macaulay property of monomial ideals by passing to squarefree monomial ideals and applying Reisner's criterion on their associated simplicial complex [29,35]. It is also used to study associated primes of monomial ideals and their powers [19,20,31].

\subsection{Our contribution}

Even though polarization has been used as a powerful tool in algebraic geometry and in applications, the inverse operation, depolarization, has been less investigated. Depolarization can be used to study the algebraic invariants of squarefree monomial ideals using general monomial ideals in less variables [34]. We note that depolarization is not unique, in the sense that a given squarefree monomial ideal might have different depolarizations.

A main goal of this paper is to find all depolarizations of a given squarefree monomial ideal and describe their structure combinatorially. This is achieved in Sect. 3, which contains our main theoretical results. More precisely, for any squarefree monomial ideal $I$, we define the so-called depolarization orders, and we show that any such order gives rise to a depolarization of $I$, see Proposition 3.5. Moreover, we show that any depolarization of $I$ can be constructed this way, see Theorem 3.7. As a given squarefree monomial ideal $I$ shares several important features with its depolarizations, the aforementioned combinatorial characterization can be used to select a convenient depolarization of $I$ in order to study the properties of either $I$ or any of its depolarizations. For example, one immediately obtains the Hilbert function of these ideals by studying only one of them, as they are closely related. It is also interesting to study the behavior of other properties and features that are not shared within the family of depolarizations of $I$. We will use such dissimilarities to identify a particular depolarization whose invariants are easier to compute and provide information about all depolarizations of I. See, e.g., Proposition 3.13.

\subsection{Applications in system reliability theory}

In applications, it is sometimes convenient to work with squarefree monomial ideals, i.e., with polarizations of monomial ideals, and use all their features as combinatorial 
objects, as seen in $[29,37]$. However, on many occasions it makes sense to work on depolarizations of squarefree monomial ideals and reduce the number of variables of their corresponding rings. See, e.g., $[3,38]$ for similar considerations in different contexts. We propose to explore both directions in the context of algebraic analysis of the reliability of systems. In previous works [14,32,36,42-46], the authors have studied the ideals associated with coherent systems for which the performing probabilities of different components are independent, and used their algebraic invariants such as Hilbert function and Betti numbers to compute the reliability of such systems. However, most of the work is devoted to binary systems whose associated ideals are squarefree. But, in practice many systems are non-binary, i.e., their components have multiple possible states, and hence, their associated ideals are not squarefree. In this paper, we use polarization tools to study the reliability of a general multistate system via binary systems, and we use depolarization as a dimension and variable reduction to study the reliability of binary systems.

\subsection{Structure of the paper}

Section 2 gives the necessary preliminaries on polarization and depolarization. In Sect. 3, we introduce the support posets as combinatorial tools to explore all depolarizations of a given squarefree monomial ideal. In Theorem 3.7, we show that every depolarization of a given monomial ideal $I$ can be obtained from its support poset. We describe the structure of depolarizations of $I$ in terms of its associated poset. This gives rise to a new bound for the projective dimension of monomial ideals, see Theorem 3.12. Moreover, we describe several families of ideals for which there exists at least one quasi-stable ideal among their depolarizations. The algebraic invariants of quasi-stable ideals are easier to compute, see, e.g., [41]. Therefore, we can compute the algebraic invariants of such ideals using their corresponding quasi-stable ideals. In Sect. 4, we turn to algebraic studies of reliability of networks and we describe how one can apply polarization and depolarization tools to compute the reliability of coherent multistate systems. Finally, we give several examples applying the depolarization tools developed through this paper for dimensional reduction in coherent systems.

\section{Polarization and depolarization}

Throughout this paper, we will assume that $R=\mathbf{k}\left[x_{1}, \ldots, x_{n}\right]$ is a polynomial ring in $n$ indeterminates over a field $\mathbf{k}$ on which we make no explicit assumptions. For any monomial ideal $I \subseteq R$, we let $G(I)=\left\{m_{1}, \ldots, m_{r}\right\}$ be the unique minimal monomial generating set of $I$.

Definition 2.1 Let $a=\left(a_{1}, \ldots, a_{n}\right)$ and $\mu=\left(b_{1}, \ldots, b_{n}\right)$ be two elements in $\mathbb{N}^{n}$ with $b_{i} \leq a_{i}$ for all $i$. The polarization of $\mu$ in $\mathbb{N}^{a_{1}+\cdots+a_{n}}$ is the multi-index

$$
\bar{\mu}=(\underbrace{1, \ldots, 1}_{b_{1}}, \underbrace{0, \ldots, 0}_{a_{1}-b_{1}}, \ldots, \underbrace{1, \ldots, 1}_{b_{n}}, \underbrace{0, \ldots, 0}_{a_{n}-b_{n}}) .
$$


The polarization of $\mathbf{x}^{\mu}=x_{1}^{b_{1}} \cdots x_{n}^{b_{n}} \in R$ with respect to $a$ is the squarefree monomial $\mathbf{x}^{\bar{\mu}}=x_{1,1} \cdots x_{1, b_{1}} \cdots x_{n, 1} \cdots x_{n, b_{n}}$ in $S=\mathbf{k}\left[x_{1,1}, \ldots, x_{1, a_{1}}, \ldots, x_{n, 1}, \ldots, x_{n, a_{n}}\right]$. Note that for ease of notation we used $\mathbf{x}$ with two different meanings in this definition. Let $I=\left\langle m_{1}, \ldots, m_{r}\right\rangle \subseteq R$ be a monomial ideal, and let $a_{i}$ be the maximum exponent to which indeterminate $x_{i}$ appears among the generators of $I$. The polarization of $I$, denoted by $I^{P}$, is the monomial ideal in $S$ given by $I^{P}=\left\langle\overline{m_{1}}, \ldots, \overline{m_{r}}\right\rangle$, where $\overline{m_{i}}$ is the polarization of $m_{i}$ with respect to $a$.

Note that Definition 2.1 is a combinatorial expression of the following result of Fröberg [12] as given in [47] in which $I^{\prime}$ is a polarization of $I$.

Proposition 2.2 For any monomial ideal $I \subset R$, there is a squarefree monomial ideal $I^{\prime} \subset R^{\prime}$ such that $R / I=R^{\prime} /\left(I^{\prime}+(\underline{h})\right)$, where $\underline{h}$ is a regular sequence on $R^{\prime} / I^{\prime}$ of forms of degree one.

Before defining the depolarization of ideals, we would like to note that as stated in Sect. 1, the polarization of monomial ideals has been extensively studied in the commutative algebra literature. Here we provide a thorough study of the reverse process, called depolarization, to provide algebraic tools to compute the reliability of large networks by reducing the number of variables and the dimension of networks.

Definition 2.3 Let $R, S$ and $T$ be polynomial rings over the field $\mathbf{k}$. Let $I \subseteq R$ be a squarefree monomial ideal. A depolarization of $I$ is a monomial ideal $J \subseteq S$ such that $I$ is isomorphic to $J^{P} \subseteq T$, that is: there is a bijective map $\varphi$ from the set of variables of $R$ to the set of variables of $T$ such that $\varphi(G(I))=G\left(J^{P}\right)$, where $G\left(J^{P}\right)$ is the unique minimal monomial generating set of $J^{P}$.

Note that the rings $R$ and $T$ above should have the same number of variables.

Example 2.4 Consider the squarefree monomial ideal $I=\langle x y z, x y t, y z t, y t u\rangle \subseteq$ $R=\mathbf{k}[x, y, z, t, u]$. The ideals $J=\left\langle a b^{2}, a^{2} b, a b c, a^{2} c\right\rangle$ and $J^{\prime}=\left\langle a b^{2}, a b c, b^{3}, b^{2} c\right\rangle$ in $S=\mathbf{k}[a, b, c]$ are two different depolarizations of $I$.

To check this, observe that $J^{P}=\left\langle a_{1} b_{1} b_{2}, a_{1} a_{2} b_{1}, a_{1} b_{1} c_{1}, a_{1} a_{2} c_{1}\right\rangle \subseteq \mathbf{k}\left[a_{1}, a_{2}, b_{1}\right.$, $\left.b_{2}, c_{1}\right]$ and we have an isomorphism between $I$ and $J^{P}$ via the correspondence $a_{1} \mapsto y, a_{2} \mapsto x, b_{1} \mapsto t, b_{2} \mapsto u, c_{1} \mapsto z$. On the other hand, $J^{\prime P}=$ $\left\langle a_{1} b_{1} b_{2}, a_{1} b_{1} c_{1}, b_{1} b_{2} b_{3}, b_{1} b_{2} c_{1}\right\rangle \subseteq \mathbf{k}\left[a_{1}, b_{1}, b_{2}, b_{3}, c_{1}\right]$ is isomorphic to $I$ by $a_{1} \mapsto$ $x, b_{1} \mapsto y, b_{2} \mapsto t, b_{3} \mapsto u, c_{1} \mapsto z$

Remark 2.5 As seen in Proposition 2.2, depolarization is a combinatorial way to perform identification of variables arisen from a regular sequence of linear forms. A natural question would be whether every such identification of variables can be read as a depolarization of the original ideal. In the following example, we show that this is not true in general. Consider the following three ideals from [38, Example 9.5]:

$$
\begin{aligned}
M & =\left\langle x_{1}^{3}, x_{2}^{2}, x_{3}^{2}, x_{1}^{2} x_{2}, x_{2}^{2} x_{3}, x_{1} x_{2} x_{3}\right\rangle \subseteq \mathbf{k}\left[x_{1}, x_{2}, x_{3}\right] \\
\mathcal{M} & =\left\langle x_{12} x_{13} x_{14}, x_{21} x_{24}, x_{31} x_{34}, x_{13} x_{14} x_{24}, x_{12} x_{14} x_{34}, x_{14} x_{24} x_{34}\right\rangle \\
& \subseteq \mathbf{k}\left[x_{12}, x_{13}, x_{14}, x_{21}, x_{24}, x_{31}, x_{34}\right]
\end{aligned}
$$




$$
\begin{aligned}
\mathcal{O} & =\left\langle x_{12} x_{13} x_{14}, x_{12} x_{24}, x_{13} x_{34}, x_{13} x_{14} x_{24}, x_{12} x_{14} x_{34}, x_{14} x_{24} x_{34}\right\rangle \\
& \subseteq \mathbf{k}\left[x_{12}, x_{13}, x_{14}, x_{24}, x_{34}\right] .
\end{aligned}
$$

Both ideals $M$ and $\mathcal{O}$ can be obtained from $\mathcal{M}$ by identifying a set of variables together. More precisely, in $M$ we relabel every variable $x_{i j}$ with the variable $x_{i}$, and in $\mathcal{O}$ we identify the following sets of variables with each other $\left\{x_{13}, x_{31}\right\}$ and $\left\{x_{12}, x_{21}\right\}$. Lemma 10.4 from [38] implies that these identifications of variables arise from a regular sequence of linear forms. However, we note that neither of them is a depolarization of $\mathcal{M}$.

\section{Combinatorics of depolarization ideals}

\subsection{The support poset}

Let $R=\mathbf{k}\left[x_{1}, \ldots, x_{n}\right]$ be a polynomial ring in $n$ variables. For any monomial $m$ of $R$ the support of $m$, denoted by $\operatorname{supp}(m)$, is defined as the set of indices of variables which divide $m$. The support of a monomial ideal $I \subseteq R$ is $\operatorname{supp}(I)=\bigcup_{m \in G(I)} \operatorname{supp}(m)$, where $G(I)$ is the unique minimal monomial generating set of $I$. We say that an ideal $I$ has full support if $\operatorname{supp}(I)=\{1, \ldots, n\}=[n]$. For ease of notation, we assume that ideals have full support, unless otherwise stated.

Let $I$ be a squarefree monomial ideal with $G(I)=\left\{m_{1}, \ldots, m_{r}\right\}$. For each $i$ in $\operatorname{supp}(I)$, we define the set $C_{i} \subseteq \operatorname{supp}(I)$ as

$$
C_{i}=\bigcap_{\substack{m \in G(I) \\ x_{i} \text { divides } m}} \operatorname{supp}(m) .
$$

In other words, $C_{i}$ is given by the indices of all the variables that appear in every minimal generator of $I$ in which $x_{i}$ is present. Let $C_{I}=\left\{C_{1}, \ldots, C_{n}\right\}$. The poset on the elements of $C_{I}$ ordered by inclusion is called the support poset of $I$ and is denoted $\operatorname{suppPos}(I)$. We define the support poset of a general monomial ideal as the support poset of its polarization obtained from Definition 2.1.

Given $n$ subsets $C_{i}$ of $[n]$, we form the poset $\left(\mathcal{C}=\left\{C_{1}, \ldots, C_{n}\right\}, \subseteq\right)$ on elements $C_{i}$ which are ordered by inclusion. Note that some $C_{i}$ can possibly be equal to $C_{j}$ for $i \neq j$. A natural question is whether for $\operatorname{such}(\mathcal{C}, \subseteq)$ we can construct a monomial ideal $I_{\mathcal{C}}$ whose support poset is $(\mathcal{C}, \subseteq)$. This question is not easy in general. See Example 3.2 (2). In the following proposition, we provide a sufficient condition to construct such ideals. Another sufficient condition will be given in Proposition 3.13.

Proposition 3.1 Let $\left(\mathcal{C}=\left\{C_{1}, \ldots, C_{n}\right\}, \subseteq\right)$ be a poset such that $\{i\} \subseteq C_{i} \subseteq[n]$ for each $i$, and if $k \in C_{i}$ and $i \in C_{j}$, then $k \in C_{j}$ for all $i, j, k$. Let $R=\mathbf{k}\left[x_{1}, \ldots, x_{n}\right]$, and let $m_{i}=\prod_{j \in C_{i}} x_{j}$ for each $i$. For any $\sigma \subseteq[n]$, let $m_{\sigma}=\operatorname{lcm}\left(m_{i} \mid i \in \sigma\right)$, and for any collection $\Sigma$ of subsets of [n], consider the monomial ideal $I_{\Sigma}=\left\langle m_{\sigma} \mid \sigma \in \Sigma\right\rangle$. Then, $(\mathcal{C}, \subseteq)$ is the support poset of $I_{\Sigma}$ if the following properties hold:

(1) $\forall i \in[n]$ there is some $\sigma \in \Sigma$ such that $x_{i} \mid m_{\sigma}$. 
(2) If $\left\{\sigma: x_{i} \mid m_{\sigma}\right\} \subseteq\left\{\sigma: x_{j} \mid m_{\sigma}\right\}$, then $C_{j} \subseteq C_{i}$.

Proof Let $\left(\mathcal{D}=\left\{D_{1}, \ldots, D_{n}\right\}, \subseteq\right)$ be the support poset of the ideal $I_{\Sigma}$. We want to show that $D_{j}=C_{j}$ for all $j \in\{1, \ldots, n\}$.

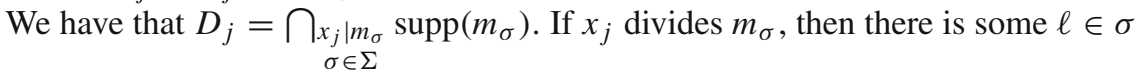
such that $x_{j}$ divides $m_{\ell}$. This implies that $m_{j}$ divides $m_{\sigma}$ for all $\sigma$ with $x_{j} \mid m_{\sigma}$. Hence, $\operatorname{supp}\left(m_{j}\right) \subset \bigcap_{x_{j} \mid m_{\sigma}} \operatorname{supp}\left(m_{\sigma}\right)$ which means $C_{j} \subseteq D_{j}$.

On the other hand, $k \in D_{j}$ implies that $x_{k}$ divides all $m_{\sigma}$, where $x_{j} \mid m_{\sigma}$. This together with condition (2) implies that $C_{k} \subseteq C_{j}$ and $k \in C_{j}$ which means $D_{j} \subseteq C_{j}$.

In the following, we show that some posets might not appear as support poset of any ideal, and on the other hand, several ideals might have the same support poset.

Example 3.2 (1) Let $C_{1}=\{1,2\}, C_{2}=\{2\}, C_{3}=\{3\}, C_{4}=\{4\}$ and $C_{5}=$ $\{4,5\}$. Let $\Sigma_{1}=\{\{1\},\{2,4\},\{3\},\{5\}\}, \Sigma_{2}=\{\{1\},\{2,3\},\{3,4\},\{5\}\}$ and $\Sigma_{3}=$ $\{\{1,3\},\{3,5\},\{1,4\},\{2,5\}\}$. These three collections satisfy the conditions in Proposition 3.1 , and hence, $\left(\mathcal{C}=\left\{C_{1}, \ldots, C_{5}\right\}, \subseteq\right)$ is the support poset of the ideals $I_{\Sigma_{1}}=\left\langle x_{1} x_{2}, x_{2} x_{4}, x_{3}, x_{4} x_{5}\right\rangle, I_{\Sigma_{2}}=\left\langle x_{1} x_{2}, x_{2} x_{3}, x_{3} x_{4}, x_{4} x_{5}\right\rangle$ and $I_{\Sigma_{3}}=\left\langle x_{1} x_{2} x_{3}, x_{3} x_{4} x_{5}, x_{1} x_{2} x_{4}, x_{2} x_{4} x_{5}\right\rangle$.

(2) Let $\mathcal{C}$ be given by $C_{1}=\{1\}, C_{2}=\{1,2\}$ and $C_{3}=\{1,2,3\}$, then there is no monomial ideal $I \subseteq R\left[x_{1}, x_{2}, x_{3}\right]$ such that $(\mathcal{C}, \subseteq)$ is the support poset of $I$. To see this, observe that $x_{1} x_{2} x_{3}$ must be one of the minimal generators of $I$, hence the only one, but $\mathcal{C}$ is not the support poset of $I=\left\langle x_{1} x_{2} x_{3}\right\rangle$.

(3) Let $C_{1}=\{1,2,4\}, C_{2}=\{1,2,4\}, C_{3}=\{1,2,3,4\}, C_{4}=\{4\}, C_{5}=$ $\{1,2,4,5,6\}, C_{6}=\{4,6\}, C_{7}=\{7\}, C_{8}=\{7,8\}, C_{9}=\{7,8,9\}, C_{10}=$ $\{7,8,10\}$. Then, for $\Sigma=\{\{3\},\{6,7\},\{5\},\{9\},\{10\}\}$, the ideal

$$
I_{\Sigma}=\left\langle x_{1} x_{2} x_{3} x_{4}, x_{4} x_{6} x_{7}, x_{1} x_{2} x_{4} x_{5} x_{6}, x_{7} x_{8} x_{9}, x_{7} x_{8} x_{10}\right\rangle \subseteq \mathbf{k}\left[x_{1}, \ldots, x_{10}\right]
$$

has $\left(\mathcal{C}=\left\{C_{1}, \ldots, C_{10}\right\}, \subseteq\right)$ as its support poset.

Remark 3.3 Note that in any support poset, $k \in C_{i}$ and $i \in C_{j}$ imply that $k \in C_{j}$. We can use this fact to visualize support posets using their Hasse diagrams, where each node is labeled by their elements which are not in any of the nodes below it.

The support poset of any monomial ideal $I \subseteq R=\mathbf{k}\left[x_{1}, \ldots, x_{n}\right]$, together with a given ordering $<$ on the variables $x_{1}, \ldots, x_{n}$, induces a partial order $\prec$ on the set of variables as follows: $x_{i} \prec x_{j}$ if $C_{i} \subset C_{j}$ or if $C_{i}=C_{j}$ and $x_{i}<x_{j}$. We call this partial order the <-support poset of $I$ and denote it by $\operatorname{suppPos}_{<}(I)$. If $C_{i} \neq C_{j}$ for every pair of indices, then $\operatorname{suppPos}(I)$ is equal to the <-support poset of $I$ for any order $<$. See Fig. 1a for Example 3.2 (1).

Note that, the Hasse diagram of $\operatorname{suppPos}_{<}(I)$ can be obtained from the Hasse diagram of suppPos $(I)$ in which every node $C$ labeled with more than one index is substituted by a vertical line of nodes labeled by distinct elements of $C$, ordered by $<$. In other words, every <-support poset of $I$ is a refinement of $\operatorname{suppPos}(I)$. See Fig. 2 as a <-support poset of $I_{\Sigma}$ in Example 3.2 (3) for any order on the variables which is compatible with $x_{1}<x_{2}$. 


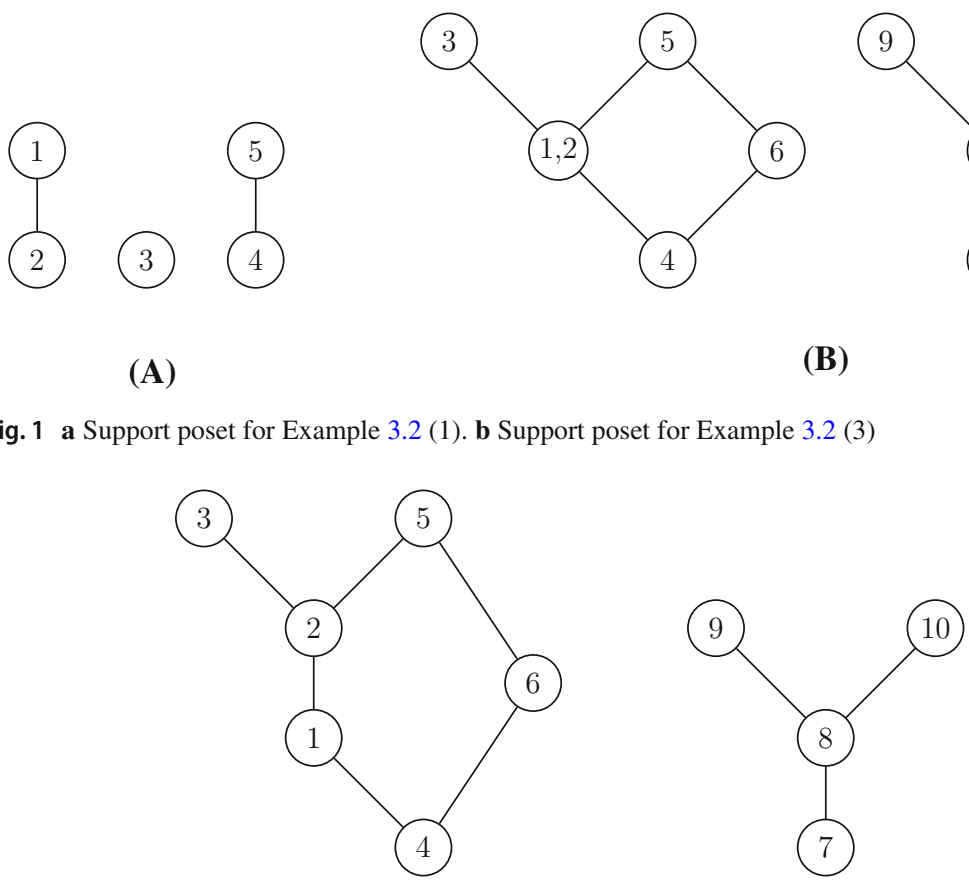

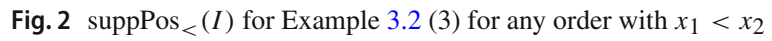

\subsection{Depolarization orders}

Recall that a subset $C$ of a poset $(\mathcal{P}, \prec)$ is a chain if any two elements of $C$ are comparable. We say that a chain $C$ of $(\mathcal{P}, \prec)$ is a path if there is no element $p \notin C$ such that $\min (C) \prec p \prec \max (C)$ and $p$ is comparable to every element in $C$. In other words, a path is a chain with no gaps, i.e., an interval within a chain. An antichain is a set of pairwise incomparable elements in $(\mathcal{P}, \prec)$.

Definition 3.4 Given an order $<$ on the variables of $R$, a depolarization order of a squarefree monomial ideal $I \subseteq R$ is a partition of $\operatorname{suppPos}_{<}(I)$ into disjoint paths.

We now show that depolarization orders characterize all depolarizations of $I$. Namely, every depolarization order gives rise to a depolarization of $I$, and every depolarization of $I$ can be realized as a depolarization obtained by such an order.

Proposition 3.5 Using any depolarization order of a squarefree monomial ideal I, we can construct a depolarization of $I$.

Proof Let $(\mathcal{P}, \prec)$ be a depolarization order for a squarefree monomial ideal $I \subseteq$ $R=\mathbf{k}\left[x_{1}, \ldots, x_{n}\right]$, where $\mathcal{P}=\left\{\sigma_{1}, \ldots, \sigma_{k}\right\}$ and each $\sigma_{i}$ is a path in $\operatorname{suppPos}_{<}(I)$ for a given order $<$ on the variables of $R$. We construct a depolarization $J$ of $I$ in a polynomial ring $S=\mathbf{k}\left[y_{1}, \ldots, y_{k}\right]$ as follows: for each monomial $m$ in $G(I)$, consider the monomial $m^{\prime}$ given by the image of $m$ under the correspondence $x_{i} \mapsto y_{j}$ for each 
Fig. 3 A path partition of suppPos $_{<}(I)$ in Example 3.2 (3) gives a depolarization order $(\mathcal{P}, \prec)$ for $I$

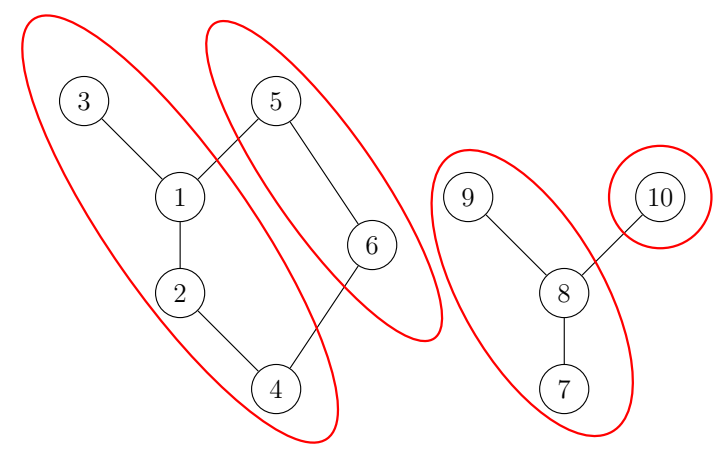

$i \in \sigma_{j}$. The monomials $m^{\prime}$ generate an ideal $J$ whose polarization $J^{P}$ is clearly equivalent to $I$ via the map sending $y_{j, \ell} \mapsto x_{\sigma_{j \ell}}$ where $\sigma_{j \ell}$ is the $\ell$ th element of $\sigma_{j}$ under the order $\prec$.

Example 3.6 The partition given by $\mathcal{P}=\{\{4,2,1,3\},\{6,5\},\{7,8,9\},\{10\}\}$ is a depolarization order for the ideal $I$ in Example 3.2 (3) for any ordering in which $x_{2}<x_{1}$. Figure 3 shows this partition.

The depolarization order $(\mathcal{P}, \prec)$ depicted in Fig. 3 gives the depolarization $J=$ $\left\langle y_{1}^{4}, y_{1} y_{2} y_{3}, y_{1}^{3} y_{2}^{2}, y_{3}^{3}, y_{3}^{2} y_{4}\right\rangle \subseteq \mathbf{k}\left[y_{1}, y_{2}, y_{3}, y_{4}\right]$ of $I$. The equivalence between $J^{P}$ and $I$ is given by $y_{1,1} \mapsto x_{4}, y_{1,2} \mapsto x_{2}, y_{1,3} \mapsto x_{1}, y_{1,4} \mapsto x_{3}, y_{2,1} \mapsto x_{6}$, $y_{2,2} \mapsto x_{5}, y_{3,1} \mapsto x_{7}, y_{3,2} \mapsto x_{8}, y_{3,3} \mapsto x_{9}, y_{4,1} \mapsto x_{10}$.

We have just seen that every depolarization order of a squarefree monomial ideal $I$ gives a depolarization of $I$. Now, we study the reverse of this process and we show that given any depolarization $J$ of $I$ we can explicitly find a depolarization order from which we can reconstruct $J$.

Theorem 3.7 Let $I=\left\langle m_{1}, \ldots, m_{r}\right\rangle \subseteq R=\mathbf{k}\left[x_{1}, \ldots, x_{n}\right]$ be a squarefree monomial ideal. Every depolarization of I can be obtained from a depolarization order of $I$.

Proof Let $J \subseteq S=\mathbf{k}\left[y_{1}, \ldots, y_{k}\right]$ be a depolarization of the ideal $I$, and let $J^{P} \subseteq$ $T=\mathbf{k}\left[y_{1,1}, \ldots, y_{1, j_{1}}, \ldots, y_{k, 1}, \ldots, y_{k, j_{k}}\right]$ be the polarization of $J$. Since $J$ is a depolarization of $I$, we know that $R$ and $T$ have the same number of variables and that $I$ and $J^{P}$ are equivalent under a map sending $x_{i}$ to $y_{a, b}$ for some $a \in\{1, \ldots, k\}$ and $b \in\left\{1, \ldots, j_{a}\right\}$. Now consider in $\{1, \ldots, n\}$ the partition $\mathcal{P}$ with $k$ subsets in which $\sigma_{i}$ contains all $j$ such that $x_{j}$ corresponds to some $y_{i, b}$ with the total order given by $j<j^{\prime}$ if $b<b^{\prime}$, where $y_{i, b} \mapsto x_{j}$ and $y_{i, b^{\prime}} \mapsto x_{j^{\prime}}$. Then, $(\mathcal{P},<)$ is a depolarization order for $I$ that produces the depolarization $J$.

Example 3.8 Consider the depolarization $J=\left\langle a b^{2}, a^{2} b, a b c, a^{2} c\right\rangle$ of the ideal $I=$ $\langle x y z, x y t, y z t, y t u\rangle$ in Example 2.4. We have that $J^{P} \subseteq \mathbf{k}\left[a_{1}, a_{2}, b_{1}, b_{2}, c_{1}\right]$ is equivalent to $I \subseteq \mathbf{k}[x, y, z, t, u]$ through the correspondence $a_{1} \mapsto y, a_{2} \mapsto x, b_{1} \mapsto t$, $b_{2} \mapsto u, c_{1} \mapsto z$. The corresponding depolarization order is $\mathcal{P}=\{\{y, x\},\{t, u\},\{z\}\}$ where the elements in the sets are given in increasing order. 


\subsection{Depolarization posets}

Let $P$ and $P^{\prime}$ be two path partitions of a given poset. We say that $P$ is a refinement of $P^{\prime}$ if for every path $C$ in $P$ there is a path $C^{\prime}$ in $P^{\prime}$ such that $C^{\prime} \subseteq C$. The set of all path partitions of a given poset are sorted by refinement and using this ordering they form themselves a poset. Let $I$ be a squarefree monomial ideal, and let $J, J^{\prime}$ be two depolarizations of $I$. We say that $J \leq J^{\prime}$ if the path partition giving rise to $J$ is a refinement of the one corresponding to $J^{\prime}$. Using this ordering, a collection of ideals that are depolarizations of a given squarefree monomial ideal $I$ forms a poset in which $I$ is the unique minimal element. We call this the depolarization poset of $I$, denoted $\mathcal{D} P(I)$. Given any monomial ideal $J$ (not necessarily squarefree), we define its depolarization poset to be the depolarization poset of its polarization $J^{P}$. In other words, $\mathcal{D} P(J):=\mathcal{D} P\left(J^{P}\right)$.

Every depolarization poset has a unique minimal element which is a squarefree monomial ideal; hence, $\mathcal{D} P(J)$ is a meet-semilattice for every monomial ideal $J$, that is, for every pair $K$ and $K^{\prime}$ in $\mathcal{D} P(J)$ there is an element in $\mathcal{D} P(J)$, denoted by $K \wedge K^{\prime}$, which is smaller than both of them. On the other hand, $\mathcal{D} P(J)$ might have several maximal elements, and therefore, it is not a lattice in general. We say that an ideal $J \subseteq \mathbf{k}\left[x_{1}, \ldots, x_{n}\right]$ is a maximum element in its depolarization poset if there is no other ideal $J^{\prime} \subseteq \mathbf{k}\left[x_{1}, \ldots, x_{m}\right]$ in $\mathcal{D} P(J)$ such that $m<n$. That means the ambient ring of $J$ has the minimal number of variables among the ambient rings of all ideals in $\mathcal{D} P(J)$.

\subsection{Copolar ideals}

Here, we study families of ideals whose algebraic invariants such as their Betti numbers are equal. A motivation for studying such family of ideals is to find some particular ideal in the family that can provide information about the rest of the ideals in the family.

Definition 3.9 Two monomial ideals $I$ and $J$ are called copolar if their polarizations are equivalent, i.e., they are in the same depolarization poset.

Copolarity is an equivalence relation in the set of monomial ideals. We say that a property or numerical invariant of an ideal is copolar if it is shared by all ideals in the same polarity class. The following proposition gives a list of copolar properties. For more details, we refer the reader to [18, Corollary 1.6.3] for items (1) to (5), to [5, Theorem 5.1] for item (6) and to [24, Corollary 4.3] for items (7) and (8).

Proposition 3.10 Let $I \subseteq S$ be a monomial ideal and let $J \subseteq T$ be its polarization. Then,

(1) $\beta_{i, j}(I)=\beta_{i, j}(J)$ for all $i$ and $j$

(2) $H_{I}(t)=(1-t)^{\delta} H_{J}(t)$ where $\delta=\operatorname{dim} T-\operatorname{dim} S$

(3) $\operatorname{height}(I)=\operatorname{height}(J)$

(4) $\operatorname{projdim}(S / I)=\operatorname{projdim}(T / J)$ and $\operatorname{reg}(S / I)=\operatorname{reg}(T / J)$

(5) $S$ / I is Cohen-Macaulay (resp. Gorenstein) if and only if T / J is Cohen-Macaulay (resp. Gorenstein). 
(6) $\lambda_{i-h, j-h}\left(S_{\mathfrak{m}} / I S_{\mathfrak{m}}\right)=\lambda_{i, j}\left(T_{\mathfrak{n}} / J T_{\mathfrak{n}}\right)$ where $\lambda_{i, j}$ 's denote the Lyubeznik numbers, $h=\operatorname{dim}(T / J)-\operatorname{dim}(S / I)$ and $\mathfrak{m}$ and $\mathfrak{n}$ are the maximal homogeneous ideals of $S$ and $T$, respectively

(7) arithmetical degree $(S / I)=$ arithmetical degree $(T / J)$

(8) The multi-complex of $I$ is shellable if and only if the simplicial complex of $J$ is shellable.

A reason behind the items in Proposition 3.10 is that the lcm-lattice [15,30] of $I$, denoted by $\operatorname{lcm}(I)$, is isomorphic to the lcm-lattice of $J$ under the map taking $\operatorname{lcm}\left(m, m^{\prime}\right)$ to $\operatorname{lcm}\left(\bar{m}, \overline{m^{\prime}}\right)$ for every pair of monomials in $G(I)$.

Lemma 3.11 Let $I$ and $J$ be two copolar ideals. Then $\operatorname{lcm}(I) \cong \operatorname{lcm}(J)$.

The lcm-lattice of a monomial ideal encodes the structure of its minimal free resolution and thus its Betti numbers [15]. In fact, polarization is a particular tool to generate ideals with isomorphic lcm-lattice. Some other important invariants are also fixed under polarization. For example, in [23] the authors proved that the Stanley conjecture can be reduced to the squarefree case via polarization, and that the Stanley projective dimension is invariant under polarization (in particular, two ideals with isomorphic $1 \mathrm{~cm}$-lattice have the same Stanley projective dimension). One recent remarkable result using polarization is given in [28], where the authors used polarization and combinatorial optimization to study the depth and regularity of powers of edge ideals of graphs and clutters.

We first take advantage of the fact that the number of variables of the ambient ring is not constant within the same polarity class, but the projective dimension is. Therefore, for any monomial ideal we can construct its depolarization poset and find the maximum elements whose ambient rings have the minimum number of variables. Since the number of variables of a polynomial ring is an upper bound for the projective dimension of its ideals, this procedure provides us with an upper bound for the projective dimension of the ideals in terms of their depolarization posets. Recall that the width of a poset is the maximum size of its antichains.

Theorem 3.12 The width of $\operatorname{suppPos}\left(I^{P}\right)$ is an upper bound for $\operatorname{projdim}(I)$.

Proof The projective dimension of $I$ is equal to the projective dimension of its polarization $I^{P}$ which is in turn the same as that of any of its depolarizations. Let $J$ be a depolarization of $I^{P}$ whose ambient ring has the smallest number of variables, say $r$. By Hilbert Syzygy Theorem, we know that $\operatorname{projdim}(J) \leq r$. By Theorem 3.7 we know that $r$ is given by the minimal number of paths in which we can partition the support poset of $I^{P}$ [observe that this number is the same for any suppPos $\operatorname{Pos}_{<}(I)$ and suppPos $(I)$ ]. Since all paths are chains, by Dilworth's Theorem [9], this number is smaller than the size of the maximal antichain of the support poset of $I^{P}$ which is the width of $\operatorname{suppPos}\left(I^{P}\right)$.

An interesting question, although out of the scope of this paper, is to compare this bound with other bounds for the projective dimension of monomial ideals, like the ones in $[7,8]$. 


\subsection{Quasi-stable ideals}

We usually study depolarization posets to find an ideal with a particularly nice property that can be transferred to its copolar ideals. For instance, here we study quasi-stable ideals [41] to compute the algebraic invariants of their copolar ideals.

Quasi-stable ideals are also called ideals of nested type [4] or ideals of Borel type [21]. A monomial ideal $I \subseteq \mathbf{k}\left[x_{1}, \ldots, x_{n}\right]$ is of nested type if each of its associated prime ideals is of form $\mathfrak{p}=\left(x_{1}, \ldots, x_{i}\right)$ for some $i$. The equivalence between these families of ideals is not immediate, and it has been proved by Seiler in [41, Proposition 4.4].

The invariants of such ideals have been extensively studied in [4,41], and it is shown that their Castelnuovo-Mumford regularity and their projective dimension can be obtained in terms of their irreducible decompositions or in terms of their Pommaret bases. Moreover, a minimal free resolution of these ideals is explicitly computed in [41, Theorem 8.6].

Therefore, if a polarity class contains a quasi-stable ideal, then we can use the aforementioned results to compute the Castelnuovo-Mumford regularity and the projective dimension of each ideal in such class. Since zero-dimensional ideals are quasi-stable, we can make use of these considerations in the polarity classes that contain at least one zero-dimensional ideal.

In the same spirit as Proposition 3.1, we provide a sufficient condition for a poset to be a support poset of a zero-dimensional monomial ideal.

Proposition 3.13 Let $n, m_{1}, \ldots, m_{n}$ be some positive integers with $1 \leq m_{i} \leq n$ for all $i$ and let $m=\sum_{i} m_{i}$. Consider a poset $(\mathcal{P}, \subseteq)$ on subsets of $\{1, \ldots, m\}$ formed by $n$ disjoint paths each of length $m_{i}$. Then, there is a squarefree monomial ideal $I$ whose support poset is $\mathcal{P}$ except if $n=2$ and $m_{1} \neq m_{2}$. Moreover, if $m_{i}>1$ for all $i$, then there is a zero-dimensional monomial ideal copolar to $I$.

Proof Let $\mathcal{P}=A_{1} \sqcup \cdots \sqcup A_{n}$ where $A_{i}=\left\{\left\{a_{i, 1}\right\},\left\{a_{i, 1}, a_{i, 2}\right\}, \ldots,\left\{a_{i, 1}, \ldots, a_{i, m_{i}}\right\}\right\}$. We assume that $n>2$ or $m_{1}=m_{2}$. The remaining case is studied in Example 3.14.

For ease of notation, we identify each variable $x_{a_{i, j}}$ with its subindex $a_{i, j}$. We can assume without loss of generality that $m_{1} \geq \cdots \geq m_{n}$.

We first construct a monomial ideal generated by the following sets of monomials:

(1) $G_{1}$ consists of the monomials $\mu_{i}=a_{i, 1} \cdots a_{i, m_{i}}$ for all $i$ with $m_{i}>1$.

(2) $G_{2}$ consists of the monomials $\mu_{i, j}=a_{i, 1} \cdots a_{i, j} b_{i, j}$ for all $i, j$ with $1<j<m_{i}$. Here, $b_{i, j}=a_{\lceil i+j-1\rceil, 1}$ where $\lceil i+j-1\rceil$ denotes $i+j-1$ modulo $n$. Note that the indices $b_{i, j}$ are pairwise distinct for each $i$, as $m_{i} \leq n$.

(3) $G_{3}=\left\{a_{i, 1} a_{i^{\prime}, 1}: a_{i, 1}, a_{i^{\prime}, 1} \in G_{3}^{\prime}\right.$ and $a_{i, 1} a_{i^{\prime}, 1} \nmid m$ for any $\left.m \in G_{1} \cup G_{2}\right\}$, where $G_{3}^{\prime}$ consists of all indices $a_{i, 1}$ for $m_{i}=1$ that appeared at most once as $b_{j, k}$ in $G_{2}$ and indices $a_{i, 1}$ for $m_{i}>1$ that never appeared as $b_{j, k}$ in $G_{2}$.

We now prove that the support poset of the ideal $I_{G} \subseteq \mathbf{k}\left[x_{a_{1,1}}, \ldots, x_{a_{n, m_{n}}}\right]$ generated by the above sets of monomials is $(\mathcal{P}, \subseteq$ ).

To see this, first observe that, by construction, the monomials in $G=G_{1} \cup G_{2} \cup G_{3}$ do not divide each other. Now we show that for each pair of variables $a_{i, j}, a_{i^{\prime}, j^{\prime}}$ with $i \neq i^{\prime}$ there is at least one monomial in $G$ which only contains one of these variables. 
If $j>1$ or $j^{\prime}>1$, then it is easy to find such a monomial in $G_{1}$. Now assume that $j=j^{\prime}=1$. If $m_{i}>1$ or $m_{i^{\prime}}>1$, then such a monomial can be found in $G_{1}$. Otherwise, they appear in separate monomials in $G_{2}$ or $G_{3}$.

Now, we show that $C_{a_{i, j}}=\left\{a_{i, 1}, \ldots, a_{i, j}\right\}$ for every variable $a_{i j}$. First note that every variable $a_{i, 1}$ appears at least once in $G_{2}$ or $G_{3}$ without the rest of the variables $a_{i, j}$ for $j>1$. Thus, $C_{a_{i, 1}}=\left\{a_{i, 1}\right\}$. For $m_{i}>1, a_{i, m_{i}}$ appears only in the monomial $\mu_{i}=a_{i, 1} \cdots a_{i, m_{i}}$ in $G_{1}$; hence, $C_{a_{i, m_{i}}}=\left\{a_{i, 1}, \ldots, a_{i, m_{i}}\right\}$. Now assume that $1<j<$ $m_{i}$. The variable $a_{i, j}$ appears always together with all the variables $a_{i, j^{\prime}}$ for $j^{\prime}<j$ since they all divide the monomials $\mu_{i}$ in $G_{1}$ and $\mu_{i, j} \in G_{2}$. On the other hand, if $m_{i}>\ell>j$, then by the construction of $G_{2}$, there is at last one monomial $\mu_{i, j}$ in which $a_{i, \ell}$ is not present. Hence, $C_{a_{i, j}}=\left\{a_{i, 1}, \ldots, a_{i, j}\right\}$ which completes the proof.

Moreover, if $m_{i}>1$ for each $i$, then using the chain partition given by the disjoint paths themselves, the corresponding depolarization of $I$ has one variable for each $i$ whose pure power appears in $G_{1}$, which implies that $I$ is zero-dimensional.

Example 3.14 Let $n=2, m_{1}=2$ and $m_{2}=1$. Then, the minimal generating set of any monomial ideal $I$ with the support poset $\mathcal{P}=\{\{1\},\{1,2\},\{3\}\}$ must include a monomial divisible by $x_{1} x_{2}$. Therefore, the only candidates for such an ideal are $\left\langle x_{1} x_{2} x_{3}\right\rangle$, $\left\langle x_{1} x_{2}, x_{3}\right\rangle,\left\langle x_{1} x_{2}, x_{1} x_{3}\right\rangle,\left\langle x_{1} x_{2}, x_{2} x_{3}\right\rangle$ and $\left\langle x_{1} x_{2}, x_{1} x_{3}, x_{2} x_{3}\right\rangle$. However, none of them has $\mathcal{P}$ as it support poset.

Example 3.15 Consider the poset $(\mathcal{P}, \subseteq)$ on the following subsets of $\{1, \ldots, 14\}$. Let $A_{1}=\{\{1\}, \ldots,\{1,2,3,4,5,6\}\}, A_{2}=\{\{7\}, \ldots,\{7,8,9,10\}\}, A_{3}=\{\{11\}\}, A_{4}=$ $\{\{12\}\}, A_{5}=\{\{13\}\}, A_{6}=\{\{14\}\}$. Then, following the notation of the proof of Proposition 3.13 we have that $(\mathcal{P}, \subseteq)$ is the support poset of the ideal generated by the monomials in $G_{1} \cup G_{2} \cup G_{3}$, where

$$
\begin{aligned}
G_{1} & =\left\{x_{1} x_{2} x_{3} x_{4} x_{5} x_{6}, x_{7} x_{8} x_{9} x_{10}\right\} \\
G_{2} & =\left\{x_{1} x_{2} x_{7}, x_{1} x_{2} x_{3} x_{11}, x_{1} x_{2} x_{3} x_{4} x_{12}, x_{1} x_{2} x_{3} x_{4} x_{5} x_{13}, x_{7} x_{8} x_{11}, x_{7} x_{8} x_{9} x_{12}\right\} \\
G_{3} & =\left\{x_{1} x_{14}, x_{13} x_{14}\right\} \text { and } G_{3}^{\prime}=\left\{x_{1}, x_{13}, x_{14}\right\} .
\end{aligned}
$$

Example 3.16 Consider the following monomial ideal in 9 variables:

$$
I=\left\langle x_{1} x_{2} x_{3} x_{4}, x_{5} x_{6} x_{7}, x_{8} x_{9}, x_{1} x_{2} x_{3} x_{5}, x_{1} x_{2} x_{8}, x_{5} x_{6} x_{8}, x_{1} x_{5} x_{8}\right\rangle
$$

By computing its associated primes, we see that $I$ is not quasi-stable. However, by determining the depolarization poset of $I$ we found that the ideal

$$
J=\left\langle y_{1}^{4}, y_{2}^{3}, y_{3}^{2}, y_{1}^{3} y_{2}, y_{1}^{2} y_{3}, y_{2}^{2} y_{3}, y_{1} y_{2} y_{3}\right\rangle \subseteq \mathbf{k}\left[y_{1}, y_{2}, y_{3}\right]
$$

is one of its maximum depolarizations. As $J$ is a zero-dimensional ideal and hence quasi-stable, by applying Theorems 8.11 and 9.2 in [41] we obtain $\operatorname{projdim}(J)=2$ and $\operatorname{reg}(J)=5$, thus obtaining these invariants for $I$. 


\subsection{Polarization and free resolutions}

We finish this section with some considerations on polarization and free resolutions. The results in this subsection will be used in Sect. 4 when we study the algebraic analysis of system reliability. First we define the polarization of a resolution. Let

$$
\mathbb{F}: \cdots \rightarrow F_{i} \stackrel{\delta_{i}}{\rightarrow} F_{i-1} \stackrel{\delta_{i-1}}{\longrightarrow} F_{i-2} \rightarrow \ldots
$$

be a multigraded chain complex of $R$-modules, i.e., $F_{i}=\bigoplus_{j=1}^{r_{i}} R\left(-\mu_{i, j}\right)$ where $\mu_{i j} \in \mathbb{N}^{n}$ and the differentials $\delta_{i}$ have multidegree 0 . The differentials $\delta_{i}$ are given by matrices $A_{i}$ whose entries are monomials in $\mathbb{N}^{n}$. We denote by $e_{i, j}$ the standard generator of the $j$ th summand of $F_{i}$ whose multidegree is $\mu_{i, j}$. Then, the $j$ th column of $A_{i}$ is given by $\left(a_{1, j}^{i}, a_{2, j}^{i}, \ldots, a_{r_{i-1}, j}^{i}\right)$ where $\delta\left(e_{i, j}\right)=\sum_{k=1}^{r_{i-1}} a_{k, j}^{i} e_{i-1, k}$ and the $e_{i-1, k}$ are the standard generators of $F_{i-1}$. The nonzero entries $a_{k, j}^{i}$ are given by $\mu_{i, j} / \mu_{i-1, k}$.

Definition 3.17 We define $\overline{\mathbb{F}}$, the polarization of $\mathbb{F}$, as the chain complex given by

$$
\overline{\mathbb{F}}: \cdots \rightarrow \bar{F}_{i} \stackrel{\bar{\delta}_{i}}{\rightarrow} \bar{F}_{i-1} \stackrel{\bar{\delta}_{i-1}}{\longrightarrow} \bar{F}_{i-2} \rightarrow \ldots
$$

where $\bar{F}_{i}=\bigoplus_{j=1}^{r_{i}} R\left(-\bar{\mu}_{i j}\right)$ if $F_{i}=\bigoplus_{j=1}^{r_{i}} R\left(-\mu_{i j}\right)$ and the matrices $\bar{A}_{i}$ of the differentials $\bar{\delta}_{i}$ are given by

$$
\bar{a}_{j, k}^{i}= \begin{cases}0 & \text { if } a_{j, k}^{i}=0 \\ x_{1, c_{1}+1} \cdots x_{1, b_{1}} \cdots x_{n, c_{n}+1} \cdots x_{n, b_{n}} & \text { if } a_{j, k}^{i} \neq 0, \mu_{i, j}=\mathbf{x}^{b}, \mu_{i-1, k}=\mathbf{x}^{c},\end{cases}
$$

where $b=\left(b_{1}, \ldots, b_{n}\right)$ and $c=\left(c_{1}, \ldots, c_{n}\right)$. Note that if $0 \neq a_{j, k}^{i} \in \mathbf{k}$, then $\bar{a}_{j, k}^{i}=a_{j, k}^{i}$.

By polarizing a resolution of a monomial ideal, we obtain a resolution of its polarization. This is a consequence of Theorem 3.3 in [15]. Here, we present our own proof to keep track of the explicit changes in each multidegree. The use of depolarization to compute resolutions of a monomial ideal from the resolutions of its polarization is also a well-known result, see Examples 3.4 in [12,15].

Proposition 3.18 Let $\mathbb{F}$ be a multigraded free resolution of a monomial ideal I. The polarization $\overline{\mathbb{F}}$ of $\mathbb{F}$ is a multigraded free resolution of the polarization of $I$. Moreover, the ranks and the graded ranks of $\overline{\mathbb{F}}$ are equal to those of $\mathbb{F}$. In the case of multigraded ranks, we have that the $(i, \mu)$-rank of $\mathbb{F}$ equals the $(i, \bar{\mu})$-rank of $\overline{\mathbb{F}}$.

Proof Given the fact that $\mathbb{F}$ is a multigraded free resolution of $I$ and the construction of $\overline{\mathbb{F}}$ by polarization, we only need to prove that $\operatorname{Im}\left(\bar{\delta}_{i}\right)=\operatorname{Ker}\left(\bar{\delta}_{i-1}\right)$.

We know that $\delta^{2}=0$. Explicitly, we have that

$$
\delta_{i-1} \delta_{i}\left(e_{i, j}\right)=\sum_{k=1}^{r_{i-1}} \sum_{l=1}^{r_{i-2}} a_{k, j}^{i} a_{l, k}^{i-1} e_{i-2, l}=0 \quad \forall i, j
$$


This implies that

$$
\sum_{k=1}^{r_{i-1}} \sum_{l=1}^{r_{i-2}} a_{k, j}^{i} a_{l, k}^{i-1}=\sum_{k=1}^{r_{i-1}} \sum_{l=1}^{r_{i-2}} \frac{\mu\left(e_{i, j}\right)}{\mu\left(e_{i-1, k}\right)} \frac{\mu\left(e_{i-1, k}\right)}{\mu\left(e_{i-2, l}\right)}=0 \quad \forall i, j .
$$

On the other hand, from the definitions of the maps in $\overline{\mathbb{F}}$ we have that for any $i, j$

$$
\bar{\delta}_{i-1} \bar{\delta}_{i}\left(\bar{e}_{i, j}\right)=\sum_{k=1}^{r_{i-1}} \sum_{l=1}^{r_{i-2}} \bar{a}_{k, j}^{i} \bar{a}_{l, k}^{i-1} \bar{e}_{i-2, l}
$$

Now by polarizing (3.1), we obtain

$$
\sum_{k=1}^{r_{i-1}} \sum_{l=1}^{r_{i-2}} \frac{\bar{\mu}\left(e_{i, j}\right)}{\bar{\mu}\left(e_{i-1, k}\right)} \frac{\bar{\mu}\left(e_{i-1, k}\right)}{\bar{\mu}\left(e_{i-2, l}\right)}=\sum_{k=1}^{r_{i-1}} \sum_{l=1}^{r_{i-2}} \bar{a}_{k, j}^{i} \bar{a}_{l, k}^{i-1}=0
$$

and hence, $\bar{\delta}_{i-1} \bar{\delta}_{i}\left(\bar{e}_{i, j}\right)=0$ for all $i, j$. Since polarization induces a multigraded isomorphism, the result follows.

Proposition 3.18 is important in our context since it allows us to use polarization in the algebraic analysis of system reliability and obtain formulas and bounds for the reliability of the system corresponding to the polarization of a given ideal. In particular, we can use the so-called Mayer-Vietoris trees as one of the main tools applied in $[42,43]$. Mayer-Vietoris trees are a way to encode the support, i.e., the collection of multidegrees of the generators of the free modules of the so-called mapping cone resolution [6,10] of a monomial ideal. We refer the reader to [40] for the definition and the basic properties of the Mayer-Vietoris trees.

\section{Multistate systems via binary systems and vice versa}

We now turn to the application of monomial ideals to multistate system analysis, which is the motivation of this work. The algebraic approach to system reliability, developed by the authors for the binary systems, gives an insight on the structure of the systems under study besides providing good computational tools to obtain reliability polynomials and bounds. In the next few examples, we show how the structure of multistate systems can be analyzed by algebraic means and that this analysis can be transferred between binary and multistate systems using polarization and depolarization.

\subsection{Multistate coherent systems}

In reliability theory $[1,2,25,33]$, a system $S$ is given by a set of components, say $n$, denoted by $c_{i}$ for $i \in\{1, \ldots, n\}$. Each $c_{i}$ can be in a discrete number of ordered states, i.e., levels of performance, $\mathcal{S}_{i}=\left(0, \ldots, m_{i}\right)$. The system itself has $m+1$ possible states $\mathcal{S}=(0, \ldots, m)$ for some integer $m$. The states of the system measure the overall 
performance of the system. In this paper, we assume that the system (respectively, the component) in state $j$ represents better performance than the system (respectively, the component) in state $i$, whenever $j>i$. We define a structure function $\phi: \mathcal{S}_{1} \times$ $\cdots \times \mathcal{S}_{n} \rightarrow \mathcal{S}$ that for each $n$-tuple of component states outputs a state of the system. We say that the system is coherent if $\phi(\mathbf{x}) \geq \phi(\mathbf{y})$ whenever $\mathbf{x}>\mathbf{y}$, which means $x_{i} \geq y_{i}$ for every $i$ and there is at least one index $i$ such that $x_{i}>y_{i}$. Conversely, $\phi(\mathbf{x}) \leq \phi(\mathbf{y})$ whenever $\mathbf{x}<\mathbf{y}$. Examples of coherent systems include electrical and transport networks, pipelines, biological and industrial systems among many others [25]. If $m_{i}=1$, then we say that component $c_{i}$ is binary. If $m=1$, then we say that the system is binary. We have therefore the following types of systems with respect to their number of states:

- If $m=1$ and $m_{i}=1$ for all $i$, we have a binary system with binary components. These are usually simply referred to as binary systems.

- If $m>1$ and $m_{i}=1$ for all $i$, we have a multistate system with binary components.

- If $m=1$ and there is at least one $i$ with $m_{i}>1$, we have a binary system with multistate components.

- If $m>1$ and there is at least one $i$ with $m_{i}>1$, we have a multistate system with multistate components.

We follow the notation in $[13,33]$. However, we allow a more general kind of system by not restricting to the case $\max (\mathcal{S}) \leq \max \left(\mathcal{S}_{i}\right)$ for all $i$. For other definitions of multistate system and a review of multistate reliability analysis, see $[27,48]$ and the references therein.

\subsection{The algebraic method in reliability analysis}

Let $S$ be a coherent system with $n$ components. Let $0<j \leq m$; we denote by $\mathcal{F}_{S, j}$ the set of tuples of components' states $\mathbf{x}$ such that $\phi(\mathbf{x}) \geq j$. The elements of $\mathcal{F}_{S, j}$ are called $j$-working states or $j$-paths of $S$. If $m=1$, then we simply speak of working states or paths. The tuples of components' states $\mathbf{x}$ with $\phi(\mathbf{x})<j$ are called $j$-failure states or $j$-cuts, respectively, failure states or cuts for $m=1$. Let $\overline{\mathcal{F}}_{S, j}$ be the set of minimal $j$-working states (minimal $j$-paths), i.e., states in $\mathcal{F}_{S, j}$ such that degradation of the performance of any component provokes that the overall performance of the system is degraded to $j^{\prime}<j$.

Now, let $R=\mathbf{k}\left[x_{1}, \ldots, x_{n}\right]$ be a polynomial ring over a field $\mathbf{k}$. Each tuple of components' states $\left(s_{1}, \ldots, s_{n}\right) \in \mathcal{S}_{1} \times \cdots \times \mathcal{S}_{n}$ corresponds to the monomial $x_{1}^{s_{1}} \cdots x_{n}^{s_{n}}$ in $R$. The coherent property of the system is equivalent to saying that the elements of $\mathcal{F}_{S, j}$ correspond to the monomials in an ideal, denoted by $I_{S, j}$ and called the $j$ reliability ideal of $S$. The unique minimal monomial generating set of $I_{S, j}$ is formed by the monomials corresponding to the elements of $\overline{\mathcal{F}}_{S, j}$ (see [42, § 2] for more details). Hence, obtaining the set of minimal $j$-paths of $S$ is equivalent to compute the minimal generating set of $I_{S, j}$.

In order to compute the $j$-reliability of $S$, i.e., the probability that the system is performing at least at level $j$, we can use the numerator of the Hilbert series of $I_{S, j}$, denoted by $H_{I_{S, j}}$. The polynomial $H_{I_{S, j}}$ gives a formula, in terms of $x_{1}, \ldots, x_{n}$ that enumerates all the monomials in $I_{S, j}$, i.e., the monomials corresponding to the states 
in $\mathcal{F}_{S, j}$. Hence, computing the (numerator of the) Hilbert series of $I_{S, j}$ provides a method to compute the $j$-reliability of $S$ by substituting $x_{i}^{a}$ by $p_{i, a}$, the probability that the component $i$ is at least performing at level $a$, as explored in [42, § 2] (for the binary case).

Often in practice it is more useful to have bounds on the $j$-reliability of $S$ rather than the complete precise formula. In order to have a formula that can be truncated at different summands to obtain bounds for the $j$-reliability in the same way that we truncate the inclusion-exclusion formula to obtain the so-called Bonferroni bounds, we need a special way to write the numerator of the Hilbert series of $I_{S, j}$. This convenient form is given by the alternating sum of the ranks in any free resolution of the ideal $I_{S, j}$. Every monomial ideal has a minimal free resolution, which provides the tightest bounds among the aforementioned ones. In general, the closer the resolution is to the minimal one, the tighter the bounds obtained, for full details see, e.g., [42, § 3].

In summary, the algebraic method for computing the $j$-reliability of a coherent system $S$ works as follows:

(1) Associate to the system $S$ its $j$-reliability ideal $I_{S, j}$.

(2) Obtain the minimal generating set of $I_{S, j}$ to get the set $\overline{\mathcal{F}}_{S, j}$.

(3) Compute the Hilbert series of $I_{S, j}$ to have the $j$-reliability of $S$.

(3') Compute any free resolution of $I_{S, j}$. The alternating sum of the ranks of this resolution gives a formula for the Hilbert series of $I_{S, j}$ i.e., the unreliability of $S$, which provides bounds by truncation at each summand.

The choice between steps (3) or ( $\left.3^{\prime}\right)$ depends on our needs. If we are only interested in computing the full reliability formula, then we can use any algorithm that computes Hilbert series in step (3). However, if we need bounds for our system reliability, then we can compute any free resolution of $I_{S, j}$ and thus perform step $\left(3^{\prime}\right)$. If the performing probabilities of different components are independent and identically distributed (i.i.d), then in points (3) and $\left(3^{\prime}\right)$ of this procedure we only need the graded version of Hilbert series and free resolutions. Otherwise, we need their multigraded version. For more details and the proofs of the results described here, we refer to $[42,45]$. To see more applications of this method in reliability analysis, we refer to [43,44,46].

We can study multistate systems via binary systems and vice versa by means of polarization and depolarization of their $j$-reliability ideals. The main reason behind this approach is that the Hilbert series and free resolutions of monomial ideals and their polarizations are related, see Proposition 3.10. For a complete application of the polarization and depolarization operations in the algebraic method, we also need the statement that the ranks of the modules in any resolution of a monomial ideal and its polarization are the same, see Proposition 3.18. When using the polarization of a $j$-reliability ideal to study the system's reliability, we have to carefully adapt the probability associated with the monomials in the new ideal. Under independence assumption, the term $x_{1}^{a_{1}} x_{2}^{a_{2}}$ contributes $\operatorname{prob}\left(c_{1} \geq a_{1}\right) \cdot \operatorname{prob}\left(c_{2} \geq a_{2}\right)$ to the reliability of the system. If independence is not assumed, then we need to individually study the probability evaluation of each monomial. In general, one needs to know the full distribution on the failure set, although the structure of the sets is distribution-free. In the case of polarization of a system reliability ideal, we have to take care of monomials that include products of the type $x_{i, 1} \cdots x_{i, k}$ which must be evaluated as $\operatorname{prob}\left(c_{i} \geq k\right)$. 


\subsection{Examples}

Our first two examples apply the algebraic method to the analysis of the reliability of multistate coherent systems. We show that our approach, using the algebraic method, can be used to analyze the reliability of such systems in an efficient and clear way. The third and fourth examples we propose will demonstrate how the depolarization method can be used to compute the reliability of various systems. In particular, in the fourth example we demonstrate how depolarizing a family of system ideals would make the reliability computations faster. Throughout this section, we assume that the performing probabilities of different components of each system are independent.

Decreasing MS $k$-out-of- $n$ system This example is taken from [22] in which the authors define generalized multistate $k$-out-of- $n$ systems (denoted MS $k$-out-of- $n$ ) as $n$-component systems with $\phi(\mathbf{x}) \geq j(j=0, \ldots, m)$, if there exists an integer value $l(j \leq l \leq m)$ such that at least $k_{l}$ components are in states at least as good as $j$. In that paper, the authors describe ad hoc methods for computing the reliability of MS $k$-out-of- $n$ systems. Different computations are proposed for the cases that the system is increasing or decreasing (which means that the sequence of $k_{l}$ is, respectively, increasing or decreasing) and also different computations need to be done if the components are identically distributed or not. For instance, Example 8 in [22] is an MS $k$-out-of-3 system with four states $(0,1,2,3)$ such that $k_{3}=2, k_{2}=2$ and $k_{1}=3$, i.e., the system is

- In state 3 or above if at least 2 components are in state 3 or above.

- In state 2 or above if at least 2 components are in state 2 or above.

- In state 1 or above if all 3 components are in state 1 or above, or if at least 2 components are in state 2 or above, or if at least 2 components are in state 3 or above.

This is called a decreasing MS $k$-out-of- $n$ system, because the sequence of $k_{l}$ is a decreasing one. In this example, the probabilities of different components are: $p_{1,0}=$ $0.1, p_{1,1}=0.2, p_{1,2}=0.3, p_{1,3}=0.4, p_{2,0}=0.1, p_{2,1}=0.2, p_{2,2}=0.2$, $p_{2,3}=0.6, p_{3,0}=0.1, p_{3,1}=0.2, p_{3,2}=0.4, p_{3,3}=0.3$. Here, we use the algebraic method to compute the probability that the system is, respectively, in states $0,1,2$ and 3 . We obtain the same exact results as in [22].

- For the system to be in state 3, there must be at least 2 components in state 3 or above $\left(k_{3}=2\right)$. Hence, the corresponding ideal is $I_{S_{3}}=\left\langle x^{3} y^{3}, x^{3} z^{3}, y^{3} z^{3}\right\rangle$. The numerator of the Hilbert series is $H_{I_{S_{3}}}=x^{3} y^{3}+x^{3} z^{3}+y^{3} z^{3}-2\left(x^{3} y^{3} z^{3}\right)$, and when substituting the probabilities, we have that the probability that the system is in state 3 or above, denoted by $R_{S, 3}$, is 0.396 , which equals the probability that the system is exactly in state 3 , denoted $r_{S, 3}$.

- The system is in state 2 or above if at least 2 components are in state 2 or above; hence, $I_{S_{2}}=\left\langle x^{2} y^{2}, x^{2} z^{2}, y^{2} z^{2}\right\rangle$. The numerator of the Hilbert series is $H_{I_{S_{2}}}=$ $x^{2} y^{2}+x^{2} z^{2}+y^{2} z^{2}-2\left(x^{2} y^{2} z^{2}\right)$ and we obtain $R_{S, 2}=0.826$ and $r_{S, 2}=R_{S, 2}-$ $R_{S, 3}=0.826-0.396=0.430$.

- Since $k_{1}=3$, the system is in state 1 or above if all 3 components are in state 1 or above or if at least 2 components are in state 2 or above, or if at 


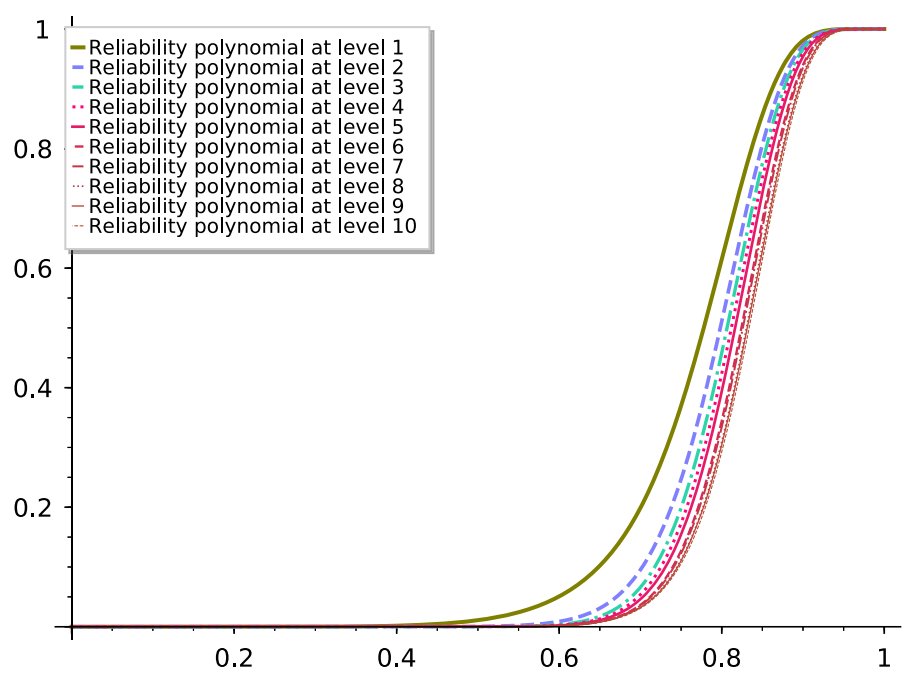

Fig. 4 Reliability polynomials at all levels for a decreasing MS $k$-out-of-10 system with $\left(k_{1}, \ldots, k_{10}\right)=$ $(9,8,7,6,5,5,4,4,3,2)$

least 2 components are in state 3 or above. The corresponding ideal is then $I_{S_{1}}=\left\langle x y z, x^{2} y^{2}, x^{2} z^{2}, y^{2} z^{2}\right\rangle$ with $H_{I_{S_{1}}}=x y z+x^{2} y^{2}+x^{2} z^{2}+y^{2} z^{2}-\left(x y^{2} z^{2}+\right.$ $x^{2} y z^{2}+x^{2} y^{2} z$ ). Thus, we obtain $R_{S, 1}=0.89$ and $r_{S, 1}=R_{S, 1}-R_{S, 2}=$ $0.89-0.826=0.064$.

- Finally, $r_{S, 0}=R_{S, 0}-R_{S, 1}=1-0.89=0.11$.

With respect to the computational applicability of the algebraic method, the authors in [22, p. 109] indicate that "For most practical engineering problems, a limited state number $M$, for example $M=10$, is big enough to describe the performances of the system and its components." We wrote a program in the computer algebra system Macaulay2 [26] to compute the Hilbert series of all the $j$-reliability ideals in a decreasing MS $k$-out-of- $n$ system. The program computes the reliability polynomials for these systems with 10 components and 10 levels of performance in less than a minute. Figure 4 shows the reliability polynomials of a $k$-out-of-10 decreasing MS system for which $k_{1}$ to $k_{10}$ are $(9,8,7,6,5,5,4,4,3,2)$. The program took 40 seconds on a laptop, ${ }^{1}$ hence, the method is practical.

Flow network A flow network $S$ has $n$ components $c_{i}, i=1, \ldots, n$, where each of them can be in $m_{i}+1$ states $\mathcal{S}_{i}=\left\{0,1, \ldots, m_{i}\right\}$ and the set of states of the system is $\mathcal{S}=\left\{0, \ldots, m_{1}+\cdots+m_{n}\right\}$. The structure function of $S$ is $\phi=x_{1}+\cdots+x_{n}$. The $j$-reliability ideal $I_{j} \subseteq R=\mathbf{k}\left[x_{1}, \ldots, x_{n}\right]$ of $S$ is generated by all monomials in $R$ of degree $j$. These ideals are stable, and therefore, the resolution given in [11] is minimal and provides a formula for the $j$-reliability of $S$ which can be truncated to obtain bounds.

The authors of [13] point out that in more complex systems, the computation of reliability bounds, denoted by $l_{\phi}^{\prime j}(\mathbf{p}), l_{\phi}^{* * j}(\mathbf{p})$ and $\tilde{l}_{\phi}^{j}(\mathbf{p})$, can become quite difficult

${ }^{1}$ CPU: intel i7-4810MQ, $2.80 \mathrm{GHz}$. RAM: $16 \mathrm{~Gb}$. 
Table 1 Structure function $\phi(\mathbf{x})$ for system $S$

\begin{tabular}{lllllllllllllllllllllllllll}
\hline$c_{1}$ & 0 & 1 & 0 & 0 & 0 & 0 & 1 & 1 & 1 & 1 & 0 & 0 & 0 & 0 & 0 & 1 & 1 & 1 & 1 & 1 & 0 & 1 & 1 & 1 \\
$c_{2}$ & 0 & 0 & 1 & 2 & 0 & 0 & 1 & 2 & 0 & 0 & 1 & 1 & 2 & 2 & 0 & 1 & 2 & 1 & 2 & 0 & 1 & 2 & 1 & 2 \\
$c_{3}$ & 0 & 0 & 0 & 0 & 1 & 0 & 0 & 0 & 1 & 0 & 1 & 0 & 1 & 0 & 1 & 1 & 1 & 0 & 0 & 1 & 1 & 1 & 1 & 1 \\
$c_{4}$ & 0 & 0 & 0 & 0 & 0 & 1 & 0 & 0 & 0 & 1 & 0 & 1 & 0 & 1 & 1 & 0 & 0 & 1 & 1 & 1 & 1 & 1 & 1 & 1 \\
$\phi(\mathbf{x})$ & 0 & 0 & 0 & 1 & 0 & 0 & 1 & 1 & 1 & 0 & 1 & 0 & 1 & 1 & 1 & 1 & 1 & 1 & 1 & 1 & 1 & 1 & 1 & 1 \\
\hline
\end{tabular}

and computationally expensive. A wide class of such complicated systems are those in which we can by some means obtain the minimal cuts or paths, but their structure is complicated. In these cases, the algebraic approach can be a very useful tool.

We consider now an example of a flow network with different levels of performance, see Example 2 in [13]. The system $S$ has two components and each of these can be in three states, $\mathcal{S}_{1}=\mathcal{S}_{2}=\{0,1,2\}$. The system itself can be in five states, $\mathcal{S}=\{0,1,2,3,4\}$. The structure function of this system is $\phi(\mathbf{x})=x_{1}+x_{2}$, i.e., the state of the system is the sum of the states of each of its components. The probability that each component is at least in state 1 is $p^{(1)}=0.9$ and the probability that each component is in state 2 is $p^{(2)}=0.8$. In [13], the authors give several bounds for the reliability of the system at level $j$. One of these bounds is based on minimal paths [denoted $\left.l_{\phi}^{\prime j}(\mathbf{p})\right]$, another one is based on minimal cuts [denoted $l_{\phi}^{* * j}(\mathbf{p})$ ], and the third one is proposed by the authors and denoted $\tilde{l}_{\phi}^{j}(\mathbf{p})$. In this case, since the components' probabilities are i.i.d., we know that $\tilde{l}_{\phi}^{j}(\mathbf{p})$ is sharp [13].

We use now the algebraic method to compute the $j$-reliabilities of this system:

- For performance at level 1 the minimal paths are $(1,0)$ and $(0,1)$, the corresponding ideal is $I_{1}=\langle x, y\rangle$ whose Hilbert function is $H_{I_{1}}=x+y-x y$. By substituting the corresponding probabilities, we have that $R_{S, 1}=0.9+0.9-0.9 \cdot 0.9=0.99$.

- For performance at level 2 the minimal paths are $(2,0),(1,1)$ and $(0,2)$, the corresponding ideal is $I_{2}=\left\langle x^{2}, x y, y^{2}\right\rangle$ whose Hilbert function is $H_{I_{2}}=x^{2}+$ $x y+y^{2}-\left(x^{2} y+x y^{2}\right)$. By substituting the corresponding probabilities, we have that $R_{S, 2}=0.8+0.9 \cdot 0.9+0.8-(0.8 \cdot 0.9+0.9 \cdot 0.8)=0.97$.

- For performance at level 3 the minimal paths are $(1,2)$ and $(2,1)$, the corresponding ideal is $I_{2}=\left\langle x^{2} y, x y^{2}\right\rangle$ whose Hilbert function is $H_{I_{2}}=x^{2} y+x y^{2}-x^{2} y^{2}$. By substituting the corresponding probabilities, we have that $R_{S, 3}=0.8 \cdot 0.9+$ $0.9 \cdot 0.8-0.8 \cdot 0.8=0.80$.

- Finally, for performance at level 4 the only minimal path is $(2,2), I_{4}=\left\langle x^{2} y^{2}\right\rangle$, $H_{I_{4}}=x^{2} y^{2}$ and we have that $R_{S, 4}=0.8 \cdot 0.8=0.64$.

Coherent system given by structure function Let $S$ be the coherent system with 4 components $c_{1}, c_{2}, c_{3}, c_{4}$ such that $c_{1}, c_{3}, c_{4}$ have two possible states 0 and 1 meaning failure and working, while $c_{2}$ has three possible states $0,1,2$. The system $S$ itself can be in two possible states, working (1) or failure (0). The probabilities $p_{i, j}$ that component $i$ is in state $j$ are: $p_{1,0}=0.2, p_{1,1}=0.8, p_{2,0}=0.3, p_{2,1}=0.2$, $p_{2,2}=0.5, p_{3,0}=0.1, p_{3,1}=0.9, p_{4,0}=0.1, p_{4,1}=0.9$. 
We want to study the reliability of $S$ whose structure function $\phi$ is given in Table 1 . One can see from the table that

$$
\overline{\mathcal{F}_{S}}=\{(1,1,0,0),(1,0,1,0),(0,2,0,0),(0,1,1,0),(0,0,1,1)\}
$$

Hence, the reliability ideal of $S$ is $I_{S}=\left\langle x y, x z, y^{2}, y z, z t\right\rangle$. The numerator of the Hilbert series of $I_{S}$ given by the alternating sum of its Betti numbers is

$$
H_{I_{S}}=x y+x z+y^{2}+y z+z t-\left(2 x y z+x y^{2}+x z t+y^{2} z+y z t\right)+x y^{2} z+x y z t .
$$

Substituting the probabilities in $H_{I_{S}}$, we obtain that $R_{S}=0.9606$. On the other hand, the polarization of $I_{S}$ is

$$
I_{S}^{P}=\left\langle x_{1} y_{1}, x_{1} z_{1}, y_{1} y_{2}, y_{1} z_{1}, z_{1} t_{1}\right\rangle \subset \mathbf{k}\left[x_{1}, y_{1}, y_{2}, z_{1}, t_{1}\right]
$$

Since $I_{S}^{P}$ is squarefree, we can use its Stanley-Reisner complex $\Delta_{I_{S}^{P}}$ to study its algebro-combinatorial features. The facets of the corresponding simplicial complex $\Delta_{I_{S}^{P}}$ are $\left\{x_{1}, y_{2}, t_{1}\right\},\left\{y_{2}, z_{1}\right\}$ and $\left\{y_{1}, t_{1}\right\}$. We can see that the numerator of the Hilbert series of $I_{S}^{P}$ is

$$
\begin{aligned}
H_{I_{S}^{P}}= & x_{1} y_{1}+x_{1} z_{1}+y_{1} y_{2}+y_{1} z_{1}+z_{1} t_{1} \\
& -\left(2 x_{1} y_{1} z_{1}+x_{1} y_{1} y_{2}+x_{1} z_{1} t_{1}+y_{1} y_{2} z_{1}+y_{1} z_{1} t_{1}\right) \\
& +x_{1} y_{1} y_{2} z_{1}+x_{1} y_{1} z_{1} t_{1}
\end{aligned}
$$

Now, we substitute the probabilities, taking into account that $y_{1} y_{2}$ corresponds to $\operatorname{prob}\left(c_{2} \geq 2\right)$. We obtain $R_{S}=0.9606$.

Studying the depolarization operation on $I_{S}^{P}$, we find that we can use the following sets for depolarizing ideal $I_{S}^{P}$

$$
\sigma_{x_{1}}=\left\{x_{1}\right\}, \quad \sigma_{y_{1}}=\left\{y_{1}\right\}, \quad \sigma_{y_{2}}=\left\{y_{1}, y_{2}\right\}, \quad \sigma_{z_{1}}=\left\{z_{1}\right\} \quad \text { and } \quad \sigma_{t_{1}}=\left\{z_{1}, t_{1}\right\} \text {. }
$$

Hence, using the partition $\left\{x_{1}\right\},\left\{y_{1}, y_{2}\right\},\left\{z_{1}, t_{1}\right\}$ we obtain a depolarization of $I_{S}^{P}$ in only three indeterminates, as $J=\left\langle a b, a c, b^{2}, b c, c^{2}\right\rangle \subset \mathbf{k}[a, b, c]$. The numerator of the Hilbert series of this ideal is

$$
H_{J}=a b+a c+b^{2}+b c+c^{2}-\left(2 a b c+a b^{2}+a c^{2}+b^{2} c+b c^{2}\right)+a b^{2} c+a b c^{2} .
$$

In order to use this expression to evaluate the reliability of $S$, we must keep track of the meaning of the new variables in terms of the ones in $I_{S}^{P}$, i.e., the monomial $b^{2}$ corresponds to $y_{1} y_{2}$ which corresponds to $\operatorname{prob}\left(c_{2} \geq 2\right)$, but $c^{2}$ corresponds to $z_{1} t_{1}$ which is evaluated as $\operatorname{prob}\left(c_{3} \geq 1\right) \cdot \operatorname{prob}\left(c_{4} \geq 1\right)$. Using these evaluations, we obtain the same result that $R_{S}=0.9606$.

Depolarization of consecutive $k$-out-of- $n$ systems and storehouse systems A consecutive k-out-of- $n: G$ system [25] is a binary system with $n$ components that works 
Table 2 Computing times for the reliability polynomials of several consecutive $k$-out-of- $n: \mathrm{G}$ system ideals and their maximal depolarizations

\begin{tabular}{llllr}
\hline$n$ & $k$ & Num. gens. & Time $J_{k, n}$ & Time $J_{k, n}^{\prime}$ \\
\hline 100 & 30 & 71 & 0.54 & 0.18 \\
100 & 15 & 86 & 34.62 & 17.81 \\
200 & 60 & 141 & 8.12 & 1.63 \\
200 & 30 & 171 & 1936.16 & 883.81 \\
300 & 90 & 211 & 56.12 & 8.63 \\
300 & 45 & 256 & OOT & 11941.60 \\
\hline
\end{tabular}

Table 3 Computing times for the reliability polynomials of several storehouse system ideals and their maximal depolarizations

\begin{tabular}{rrrllr}
\hline$n$ & $m$ & $k$ & Num. gens. & Time $I_{n, m, k}$ & Time $I_{n, m, k}^{\prime}$ \\
\hline 3 & 40 & 50 & 1161 & 0.82 & 0.17 \\
3 & 90 & 100 & 4986 & 33.92 & 2.13 \\
3 & 140 & 150 & 11311 & 282.33 & 13.91 \\
4 & 30 & 40 & 11461 & 111.49 & 14.40 \\
4 & 50 & 60 & 38831 & 1670.39 & 404.09 \\
4 & 70 & 80 & 91001 & OOT & 2697.49 \\
\hline
\end{tabular}

whenever $k$ consecutive components work. The reliability ideal of such a system is $J_{k, n}=\left\langle x_{1} \cdots x_{k}, \ldots, x_{n-k+1} \cdots x_{n}\right\rangle \subseteq \mathbf{k}\left[x_{1}, \ldots, x_{n}\right]$. The ideal $J_{k, n}$ has $n-k+1$ generators, all of degree $k$ in $n$ variables, which are corresponding to the set of all $k$-paths of the line graph [16]. The depolarization poset of $J_{k, n}$ has a maximal element $J_{k, n}^{\prime}$ whose ambient ring has $n+2-2 k$ variables, and we can use it to compute the reliability of consecutive $k$-out-of- $n:$ G systems when $n$ is large.

A storehouse system is a system with components of $n$ types ( $m$ components of each type) such that within each type, components are sorted so that the $i$ th component can be working only if the previous ones are working. The system works whenever at least $k$ components are working (with $k>m$ ). These systems can be used to model storehouses with shared capacities, industrial straps and pipelines, for instance. We denote $I_{n, m, k}$ for the associated ideals of such systems which are studies in [39, Chapter 3]. These ideals are squarefree monomial ideals in $\mathrm{nm}$ variables. The depolarization poset of the ideal $I_{n, m, k}$ has a maximal element $I_{n, m, k}^{\prime}$ whose ambient ring has only $n$ variables, and the ideal $I_{n, m, k}^{\prime}$ is generated by all monomials of degree $k$ in which each variable appears with a degree less than or equal to $m$.

Tables 2 and 3 show the timings of an algorithm implemented by the authors using the Hilbert series implementation in [26] to compute the reliability polynomial of several large consecutive $k$-out-of- $n$ systems and storehouse systems, respectively. The last two columns in each table show the times used to compute the reliability polynomial using the original squarefree ideal $J_{k, n}$, resp. $I_{n, m, k}$, and the times used to compute the reliability polynomial using the maximal depolarization $J_{k, n}^{\prime}$, resp. $I_{n, m, k}^{\prime}$. The times are in seconds. OOT means the computation was manually stopped after 24 h. Observe that the times are reduced due to the reduction in the number of variables in the ambient ring. Working with the maximal depolarization makes it possible to 
handle bigger cases that are not possible to deal with using the squarefree reliability ideals.

Acknowledgements The authors are partially funded by Grant MTM2017-88804-P of Ministerio de Economía, Industria y Competitividad (Spain). The first author was partially supported by the EPSRC Early Career Fellowship EP/R023379/1. We are grateful to the anonymous referees for very helpful comments on the earlier version of this paper.

\section{References}

1. Boedigheimer, R.A., Kapur, K.C.: Customer-driven reliability models for multistate coherent systems. IEEE Trans. Reliab. 43(1), 46-50 (1994)

2. Barlow, R.E., Proschan, F.: Statistical Theory of Reliability and Life Testing. Holt, Rinehart and Wiston, New York (1975)

3. Berkesch, C., Matusevich, L.F.: A-graded methods for monomial ideals. J. Algebra 322, 2886-2904 (2009)

4. Bermejo, I., Gimenez, P.: Saturation and Castelnuovo-Mumford regularity. J. Algebra 303, 592-617 (2006)

5. Banerjee, A., Núñez-Betancourt, L., Yanagawa, K.: Properties of Lyubeznik numbers under localization and polarization. J. Pure Appl. Algebra 219, 4872-4888 (2015)

6. Chalambous, H., Evans, E.G.: Resolutions obtained as iterated mapping cones. J. Algebra 176, 750-754 (1995)

7. Dao, H., Huneke, C., Schweig, J.: Bounds on the regularity and projective dimension of ideals associated to graphs. J. Algebraic Combin. 38(1), 37-55 (2013)

8. Dao, H., Schweig, J.: Bounding the projective dimension of a squarefree monomial ideal via domination in clutters. Proc. Amer. Math. Soc. 143, 555-565 (2015)

9. Dilworth, R.P.: A decomposition theorem for partially ordered sets. Ann. Math. 51(1), 161-166 (1950)

10. Dochtermann, A., Mohammadi, F.: Cellular resolutions from mapping cones. J. Combin. Theory Ser. A 128, 180-206 (2014)

11. Eliahou, S., Kervaire, M.: Minimal resolutions of some monomial ideals. J. Algebra 129, 1-25 (1990)

12. Fröberg, R.: A study of graded extremal rings and of monomial rings. Math. Scand. 51, 22-34 (1982)

13. Gasemir, J., Natvig, B.: Improved availability bounds for binary and multi-state systems with independent component processes. J. Appl. Probab. 54(3), 750-762 (2017)

14. Giglio, B., Wynn, H.P.: Monomial ideals and the scarf complex for coherent systems in reliability theory. Ann. Stat. 32, 1289-1311 (2004)

15. Gasharov, V., Peeva, I., Welker, V.: The lcm-lattice in monomial resolutions. Math. Res. Lett. 6, 521532 (1999)

16. He, J., Van-Tuyl, A.: Algebraic properties of the path ideal of a tree. Comm. Algebra 38(5), 1725-1742 (2010)

17. Hartshorne, R.: Connectedness of the Hilbert scheme. Inst. Hautes Etudes Sci. Publ. Math. 29, 5-48 (1966)

18. Herzog, J., Hibi, T.: Monomial Ideals. Graduate Texts in Mathematics, vol. 260. Springer, London (2010)

19. Hà, H.T., Morey, S.: Embedded associated primes of powers of squarefree monomial ideals. J. Pure Appl. Algebra 214(4), 301-308 (2010)

20. Herzog, J., Hibi, T., Qureshi, A.A.: Polarization of Koszul cycles with applications to powers of edge ideals of whisker graphs. Proc. Amer. Math. Soc. 143(7), 2767-2778 (2015)

21. Herzog, J., Popescu, D., Vladoiu, M.: On the Ext-modules of ideals of Borel type. In: Commutative Algebra, Contemp. Math. vol. 331, pp. 171-186. Amer. Math. Soc., Providence (2003)

22. Huang, J., Zuo, M.J., Wu, Y.: Generalized multi-state k-out-of-n: G systems. IEEE Trans. Reliab. 49(1), 105-111 (2000)

23. Ichim, B., Katthän, L., Moyano-Fernández, J.J.: The behavior of Stanley depth under polarization. J. Combin. Theory Ser. A 135, 332-347 (2015)

24. Jahan, A.S.: Prime filtrations of monomial ideals and polarizations. J. Algebra 312(2), 1011-1032 (2007) 
25. Kuo, W., Zuo, M.J.: Optimal Reliability Modelling. Wiley, Hoboken (2003)

26. Grayson, D.R., Stillman, M.E.: Macaulay2, a software system for research in algebraic geometry. http://www.math.uiuc.edu/Macaulay2/

27. Lisnianski, A., Levitin, G.: Multi-State System Reliability: Assesment, Optimization and Applications. World Scientific, Singapore (2003)

28. Martínez-Bernal, J., Morey, S., Villarreal, R.H., Vivares, C.E.: Depth and regularity of monomial ideals via polarization and combinatorial optimization. Acta Math. Vietnam. 44(1), 243-268 (2019)

29. Miller, E.: Topological Cohen-Macaulay criteria for monomial ideals. In: Ene V., Miller E. (eds.) Combinatorial Aspects of Commutative Algebra Contemporary Math, vol. 502, pp. 137-155 (2009)

30. Miller, E., Sturmfels, B.: Combinatorial Commutative Algebra. Springer, New York (2004)

31. Martínez-Bernal, J., Morey, S., Villarreal, R.H.: Associated primes of powers of edge ideals. Collect. Math. 63(3), 361-374 (2012)

32. Mohammadi, F.: Divisors on graphs, orientations, syzygies, and system reliability. J. Algebraic Combin. 43(2), 465-483 (2016)

33. Natvig, B.: Multi-State System Reliability Theory with Applications. Wiley, Chichester (2011)

34. Phan, J.: Properties of Monomial Ideals and Their Free Resolutions. Ph.D. thesis, Columbia University (2006)

35. Stanley, R.: Combinatorics and Commutative Algebra. Progress in Mathematics, vol. 41. Birkhäuser, Boston (1983)

36. Mohammadi, F., Sáenz-de-Cabezón, E., Wynn, H.P.: Types of signature analysis in reliability based on Hilbert series. J. Symbolic Comput. 79, 140-155 (2017)

37. Mohammadi, F.: Combinatorial and geometric view of the system reliability theory. In: International Congress on Mathematical Software, pp. 148-153. Springer International Publishing (2016)

38. Mohammadi, F., Shokrieh, F.: Divisors on graphs, binomial and monomial ideals, and cellular resolutions. Math. Z. 283(1-2), 59-102 (2016)

39. Sáenz-de-Cabezón, E.: Combinatorial Koszul homology: computations and applications. Ph.D. Thesis, Universidad de La Rioja (2008)

40. Sáenz-de-Cabezón, E.: Multigraded Betti numbers without computing minimal free resolutions. Appl. Algebra Eng. Comm. Comput. 20, 481-495 (2009)

41. Seiler, W.M.: A combinatorial approach to involution and delta-regularity II: structure analysis of polynomial modules with Pommaret bases. Appl. Algebra Eng. Comm. Comput. 20, 261-338 (2009)

42. Sáenz-de-Cabezón, E., Wynn, H.P.: Betti numbers and minimal free resolutions for multi-state system reliability bounds. J. Symbolic Comput. 44, 1311-1325 (2009)

43. Sáenz-de-Cabezón, E., Wynn, H.P.: Mincut ideals of two-terminal networks. Appl. Algebra Eng. Comm. Comput. 21, 443-457 (2010)

44. Sáenz-de-Cabezón, E., Wynn, H.P.: Computational algebraic algorithms for the reliability of generalized $k$-out-of- $n$ and related systems. Math. Comput. Simulation 82, 68-78 (2011)

45. Sáenz-de-Cabezón, E., Wynn, H.P.: Algebraic reliability based on monomial ideals: a review. In: Harmony of Gröbner Basis and The Modern Industrial Society, pp. 314-335. Wiley (2012)

46. Sáenz-de-Cabezón, E., Wynn, H.P.: Hilbert functions for design in reliability. IEEE Trans. Reliab. 64(1), 83-93 (2015)

47. Villarreal, R.H.: Monomial Algebras, Monographs and Textbooks in Pure and Applied Mathematics, vol. 238. Marcel Dekker, New York (2001)

48. Yingkui, G., Jing, L.: Multi-state system reliability: a new and systematic review. Procedia Eng. 29, $531-536(2012)$

Publisher's Note Springer Nature remains neutral with regard to jurisdictional claims in published maps and institutional affiliations. 